\title{
Comparative Study of the Reduction Process of Different Ring Structured Nitroxyl Spin Probes: An Electron Spin Resonance Study
}

\author{
V. Meenakumari ${ }^{1}$, A. Jawahar ${ }^{2}$, A. Milton Franklin Benial ${ }^{1, *}$ \\ ${ }^{1}$ Department of Physics, NMSSVN College, Nagamalai, Madurai, Tamilnadu, India \\ ${ }^{2}$ Department of Chemistry, NMSSVN College, Nagamalai, Madurai, Tamilnadu, India \\ Email address: \\ meenakumariv78@gmail.com (V. Meenakumari), ajanthajawahar@yahoo.com (A. Jawahar), miltonfranklin@yahoo.com (A. M. F. Benial) \\ ${ }^{*}$ Corresponding author
}

\section{To cite this article:}

V. Meenakumari, A. Jawahar, A. Milton Franklin Benial. Comparative Study of the Reduction Process of Different Ring Structured Nitroxyl Spin Probes: An Electron Spin Resonance Study. European Journal of Biophysics. Vol. 4, No. 2, 2016, pp. 8-15.

doi: 10.11648/j.ejb.20160402.11

Received: March 23, 2016; Accepted: April 1, 2016; Published: April 26, 2016

\begin{abstract}
Electron spin resonance spectroscopy (ESR) studies on the reduction process of nitroxyl spin probes were carried out for $1 \mathrm{mM}$ concentration of ${ }^{14} \mathrm{~N}$-labeled pyrrolidine nitroxyl spin probes, 3-carbamoyl-2,2,5,5-tetramethyl-pyrrolidine-1-oxyl

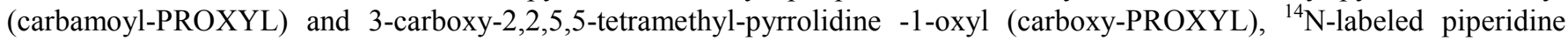
nitroxyl spin probes, 4-methoxy-2,2,6,6-tetramethyl-piperidine-1-oxyl (methoxy-TEMPO) and 4-acetamido-2,2,6,6-tetramethyl-piperidine-1-oxyl (acetamido-TEMPO) in $1 \mathrm{mM}$ concentration of ascorbic acid as a function of time. The half-life time and decay rate were estimated. The piperidine nitroxyl spin probes show a short half-life time compared with that of pyrrolidine nitroxyl spin probes. This result indicates that the higher stability of pyrrolidine nitroxyl spin probes. The ESR was also recorded for $1 \mathrm{mM}$ concentration of pyrrolidine nitroxyl spin probes and piperidine nitroxyl spin probes in pure water using X-band ESR spectrometer. The ESR parameters such as line width, hyperfine coupling constant, g-factor, signal intensity ratio and rotational correlation time were determined. These results indicate that the pyrrolidine nitroxyl spin probes have narrow line width and fast tumbling compared with the piperidine nitroxyl spin probes. Therefore, this study reveals that the pyrrolidine nitroxyl spin probes can act as good redox sensitive spin probes for free radical imaging.
\end{abstract}

Keywords: Electron Spin Resonances (ESR), Nitroxyl Spin Probe, Ascorbic Acid, Half Life Time, Decay Rate

\section{Introduction}

The nitroxyl radicals are well-known stable organic radicals. Most frequently used nitroxyl radicals invivo are piperidine and pyrrolidine classes. The stability of nitroxyl radicals is attributed to the three $\pi$-bonding system with a delocalized unpaired electron on the $\mathrm{N}-\mathrm{O}$ bond. These nitroxyl radicals are less toxic, and thereby preferable for invivo applications. Nitroxyl radicals are susceptible to oxygen concentration [1], reactive oxygen species (ROS) [2-3], and biological redox systems [4-8], and are widely used as probes for invivo electron spin resonance (ESR) measurement [9-11]. The most active use of invivo ESR spectroscopy and its imaging has been in the measurement of redox status, radical generation and partial pressure of oxygen. ESR spectroscopy has been employed in invitro redox research studies $[12,13]$. In the ESR X-band frequency the magnetic interactions of nitroxide spin labels are extremely sensitive to motion on the nanosecond time scale, which is relevant to the dynamics of bio-molecules [14].

The reduction of nitroxyl radicals by cell is primarily intracellular [15]. Ascorbic acid plays a significant role in the nitroxyl radical reduction in erythrocytes, hepatocytes, and kidney cells, which are rich in this compounds [16, 17]. Ascorbic acid is one of the primary reducing agent in biochemistry, which is directly involved in many biochemical processes, such as metabolic-oxidation-reduction, carcinogensis, and aging. The paramagnetic nitroxyl radicals could be reduced to diamagnetic hydroxylamine by 
antioxidants, such as ascorbic acid (vitamine C) or enzymatic system, with a loss of ESR signal and thus serves as a reduction sensor. However, the diamagnetic hydroxylamine could be reconverted via oxygenation to paramagnetic nitroxyl radicals with an appearance of ESR signal and thus serves as an oxidation sensor. The rate constant of both processes could be used for evaluation of reduction / oxidation balance in cells and tissues, using ESR imaging or magnetic resonance imaging (MRI) [18]. The mechanism of reduction in invivo experiments depends on several factors including the structure of the nitroxyl radical, oxygen concentration, membrane permeability, extracellular reduction by ascorbate, and possible reoxidation of the reduced form by nitroxyl radicals. The redox properties of nitroxyl radicals can give us double benefits which are (i) as redox-sensitive contrast agents and (ii) as a normal tissue-selective radio-protector. Both benefits are useful for a first diagnosis of a tumor and radiation therapy planning. These will give a radio-protective treatment for normal tissues in subsequent radiotherapy. Therefore, the magnetic resonance based redox imaging techniques using nitroxyl contrast agents can have wide possibility.

Earlier studies have shown that a cell-permeable nitroxide was reduced faster in tumor than in normal tissue [19, 20]. Therefore, monitoring the rate of transformation of suitable nitroxides to the corresponding diamagnetic species using MRI can provide invivo assessment of redox status in a noninvasive manner. Utsumi et al. obtained the simultaneous molecular imaging of reduction and oxidation processes monitored by overhauser magnetic resonance imaging (OMRI) with ${ }^{14} \mathrm{~N}$-and ${ }^{15} \mathrm{~N}$-labeled nitroxyl radicals [21]. Benial et al. reported that the permeability studies of redox sensitive nitroxyl spin probes through lipid membranes using an L-band ESR spectrometer [22]. Kinoshita et al. also reported the synthesis of tetraethyl-substitued nitroxyl radicals, which exhibit remarkable resistivity towards ascorbic acid reduction during invivo testing [23].

Recently, the stable nitroxyl radicals have been used as redox sensitive probe for invivo ESR and OMRI techniques [24-34]. In order to understand the reduction process and find the suitable nitroxyl spin probe among the pyrrolidine and piperidine nitroxyl radicals for invivo/invitro ESR and OMRI techniques. Here, we report the ESR spectroscopy studies on the kinetics and reduction mechanism of $1 \mathrm{mM}$ concentration of ${ }^{14} \mathrm{~N}$-labeled carbamoyl-PROXYL, carboxy-PROXYL, methoxy-TEMPO and acetamido-TEMPO in pure water and 1 $\mathrm{mM}$ concentration of ascorbic acid.

\section{Materials and Methods}

\subsection{Chemicals}

The spin probes, 3-carbamoyl-2,2,5,5-tetramethylpyrrolidine-1-oxyl (carbamoyl-PROXYL), 3-carboxy -2,2,5,5-tetramethyl-pyrrolidine-1-oxyl-(carboxy-PROXYL), 4-methoxy-2,2,6,6-tetramethyl-piperidine-1-oxyl (methoxy-TEMPO), 4-acetamido-2,2,6,6-tetramethyl- piperidine-1-oxyl (acetamido-TEMPO) and ascorbic acid were purchased from Sigma Aldrich Chemical Co, St. Louis, MO, USA. Deionized water (deionization by the Milli-Q system) was used for this experiment.

\subsection{ESR Measurements}

ESR spectra were recorded for $1 \mathrm{mM}$ concentration of ${ }^{14} \mathrm{~N}$-labeled carbamoyl-PROXYL, carboxy-PROXYL, methoxy-TEMPO and acetamido-TEMPO in pure water and $1 \mathrm{mM}$ concentration of ascorbic acid as a function of time using a Bruker EMS plus X-band ESR spectrometer by varying the magnetic field, 342-352 $\mathrm{mT}$; with modulation frequency, $100 \mathrm{kHz}$; field modulation amplitude, $0.2 \mathrm{mT}$; conversion time, $10 \mathrm{~ms}$; radio-frequency power, $2 \mathrm{~mW}$; receiver gain, 1000; sweep width, $10 \mathrm{mT}$; sweep time, $10 \mathrm{~s}$; point field resolution, $1024 \mathrm{k}$; and microwave frequency, 9.86 GHz. The ESR spectra were recorded in the first derivative mode at $27^{\circ} \mathrm{C}$. The temperature was controlled using a controller with water as a coolant. The ESR line width measurements were carried out for $1 \mathrm{mM}$ concentration of nitroxyl spin probes in pure water with an accuracy of $\sim \pm 0.5 \mu \mathrm{T}$.

\section{Results and Discussion}

The schematic diagram of the reduction process of nitroxyl spin probe is shown in Figure 1. The ESR spectra of $1 \mathrm{mM}$ concentration of ${ }^{14} \mathrm{~N}$-labeled carbamoyl- PROXYL, carboxy-PROXYL, methoxy-TEMPO and acetamido-TEMPO in pure water and $1 \mathrm{mM}$ concentration of ascorbic acid as a function of time are shown in Figures 2-5. The signal intensity and scavenging activity of all nitroxyl spin probes in pure water and $1 \mathrm{mM}$ concentration of ascorbic acid at various time intervals are shown in Figures 6-7. The ring structures and abbreviations of nitroxyl spin probes are shown in Table 1. The ESR parameters such as line width, hyperfine coupling constant, g-factor, signal intensity ratio and rotational correlation time for $1 \mathrm{mM}$ concentration of ${ }^{14} \mathrm{~N}$-labeled carbamoyl- PROXYL, carboxy-PROXYL, methoxy-TEMPO and acetamido-TEMPO in pure water are listed in Table 2. The half life time and decay rate of $1 \mathrm{mM}$ concentration of ${ }^{14} \mathrm{~N}$-labeled carbamoyl-PROXYL, carboxy-PROXYL, methoxy-TEMPO and acetamido-TEMPO in $1 \mathrm{mM}$ concentration of ascorbic acid are listed in Table 3.

Table 1. The Ring structures and abbreviations of nitoxyl spin probes.

\begin{tabular}{lll}
\hline Basic structure & Substituents (R) & Abbreviations \\
\hline & $2 \mathrm{CONH}_{2}$ & carbamoyl-PROXYL \\
$\mathrm{OCH}_{3}$ & methoxy-TEMPO \\
\hline
\end{tabular}


<smiles>[R]C1CC(C)(C)N([O])C1(C)C</smiles>

Nitroxyl Radical ESR detectable<smiles>[R]C1CC(C)(C)N([O])C(C)(C)C1</smiles>

Nitroxyl Radical ESR detectable

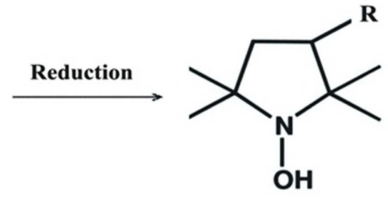

Hydroxylamine ESR Silent
Figure 1. Schematic diagram of the reduction process of nitroxyl spin probes, $R$ represents substituents.

\subsection{Line Width}

The ESR line width values for $1 \mathrm{mM}$ concentration of ${ }^{14} \mathrm{~N}$-labeled carbamoyl-PROXYL, carboxy-PROXYL, methoxy-TEMPO and acetamido-TEMPO in pure water were calculated and listed in Table 2. The line width broadening is due to the dipolar and spin exchange interactions of agent concentrations. The line width broadening is due to the interaction between the subtituents $(\mathrm{R})$ in pyrrolidine, piperidine nitroxyl spin probes and water proton. These results are in good agreement with the previous study [35-37]. The pyrrolidine nitroxyl spin probes viz., carbamoyl-PROXYL and carboxy-PROXYL have a narrow line width compared with the piperidine nitroxyl spin probes viz., methoxy-TEMPO and acetamido-TEMPO. The narrow line width values of pyrrolidine nitroxyl spin probes indicate that the interaction between the carbamoyl group/carboxyl group of pyrrolidine nitroxyl spin probes and water proton is less, but the interaction between the methyl group of piperidine nitroxyl spin probes and water proton is more. The observed line width value is $\sim 42 \%$ higher for piperidine nitroxyl spin probes compared with the pyrrolidine nitroxyl spin probes.

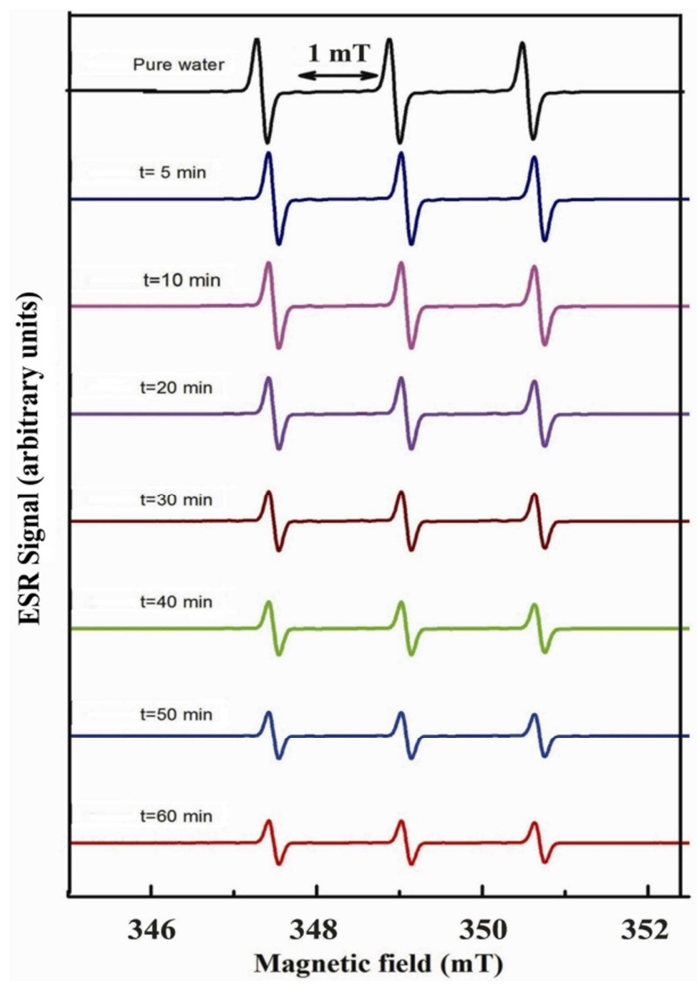

Figure 2. ESR spectra of $1 \mathrm{mM}$ concentration of ${ }^{14} \mathrm{~N}$-labeled carbamoyl-PROXYL in pure water and $1 \mathrm{mM}$ concentration of ascorbic acid as a function of time ( $t$.

Table 2. Electron spin resonance parameters for $1 \mathrm{mM}$ concentration of ${ }^{14} \mathrm{~N}$-labeled carbamoyl-PROXYL, carboxy-PROXYL, methoxy-TEMPO and acetamido-TEMPO in pure water

\begin{tabular}{|c|c|c|c|c|c|}
\hline Sample & Line width $\Delta \mathrm{B}(\mu \mathrm{T})$ & $\begin{array}{l}\text { Hyperfine coupling } \\
\text { constant }(\mathrm{mT})\end{array}$ & g-factor & $\begin{array}{l}\text { Signal intensity ratio } \\
\left(\mathbf{h}_{0} / \mathbf{h}_{-1}\right)\end{array}$ & $\begin{array}{l}\text { Rotational correlation time } \\
\tau_{\mathrm{R}}(\mathrm{s})\left(\times 10^{-11}\right)\end{array}$ \\
\hline carbamoyl-PROXYL & 117.30 & 1.603 & 2.0117 & 1.0842 & 3.145 \\
\hline carboxy-PROXYL & 119.03 & 1.603 & 2.0118 & 1.0879 & 3.336 \\
\hline methoxy-TEMPO & 166.07 & 1.701 & 2.0103 & 1.0675 & 3.584 \\
\hline acetamido-TEMPO & 166.18 & 1.706 & 2.0109 & 1.0931 & 4.918 \\
\hline
\end{tabular}

\subsection{Hyperfine Coupling Constant and g-factor}

The Hyperfine coupling constant and g-factor values for 1 $\mathrm{mM}$ concentration of ${ }^{14} \mathrm{~N}$-labeled carbamoyl-PROXYL, carboxy-PROXYL, methoxy-TEMPO and acetamido-TEMPO in pure water are listed in Table 2. The observed hyperfine coupling constant is less for pyrrolidine nitroxyl spin probes compared with piperidine nitroxyl spin probes. This shows that the Fermi contact interaction is less for pyrrolidine nitroxyl spin probes viz., carbamoyl-PROXYL and carboxy-PROXYL compared with piperidine nitroxyl spin probes viz., methoxy-TEMPO and acetamido-TEMPO. The hyperfine coupling constant of the nitroxyl spin probes agree well with the previous study [35-37]. The obtained $\mathrm{g}$-factor value confirms the isotropic nature of the system.

\subsection{Signal Intensity Ratio}

The ESR signal intensity ratio for $1 \mathrm{mM}$ concentration of ${ }^{14} \mathrm{~N}$-labeled carbamoyl-PROXYL, carboxy-PROXYL, methoxy-TEMPO and acetamido-TEMPO in pure water are listed in Table 2. The ESR signal intensity ratio becomes the unity for both pyrrolidine and piperidine nitroxyl spin probes in pure water, which reveals that the homogenous nature of the samples. These results agree well with the reported values [35-37]. 
Table 3. The half life time and decay rate of ${ }^{14} \mathrm{~N}$-labeled carbamoyl PROXYL, carboxy-PROXYL, methoxy-TEMPO and acetamido-TEMPO in $1 \mathrm{mM}$ concentration of ascorbic acid

\begin{tabular}{lll}
\hline Sample & $\begin{array}{l}\text { Half life time } \mathbf{t}_{\mathbf{1} / \mathbf{2}} \\
(\mathbf{m i n})\end{array}$ & $\begin{array}{l}\text { Signal decay rate } \\
\mathbf{1} / \boldsymbol{\tau}\left(\mathbf{m i n}^{-1}\right)\end{array}$ \\
\hline carbamoyl-PROXYL & 17.801 & 0.039 \\
carboxy-PROXYL & 13.353 & 0.052 \\
methoxy-TEMPO & 4.937 & 0.140 \\
acetamido-TEMPO & 2.644 & 0.262 \\
\hline
\end{tabular}

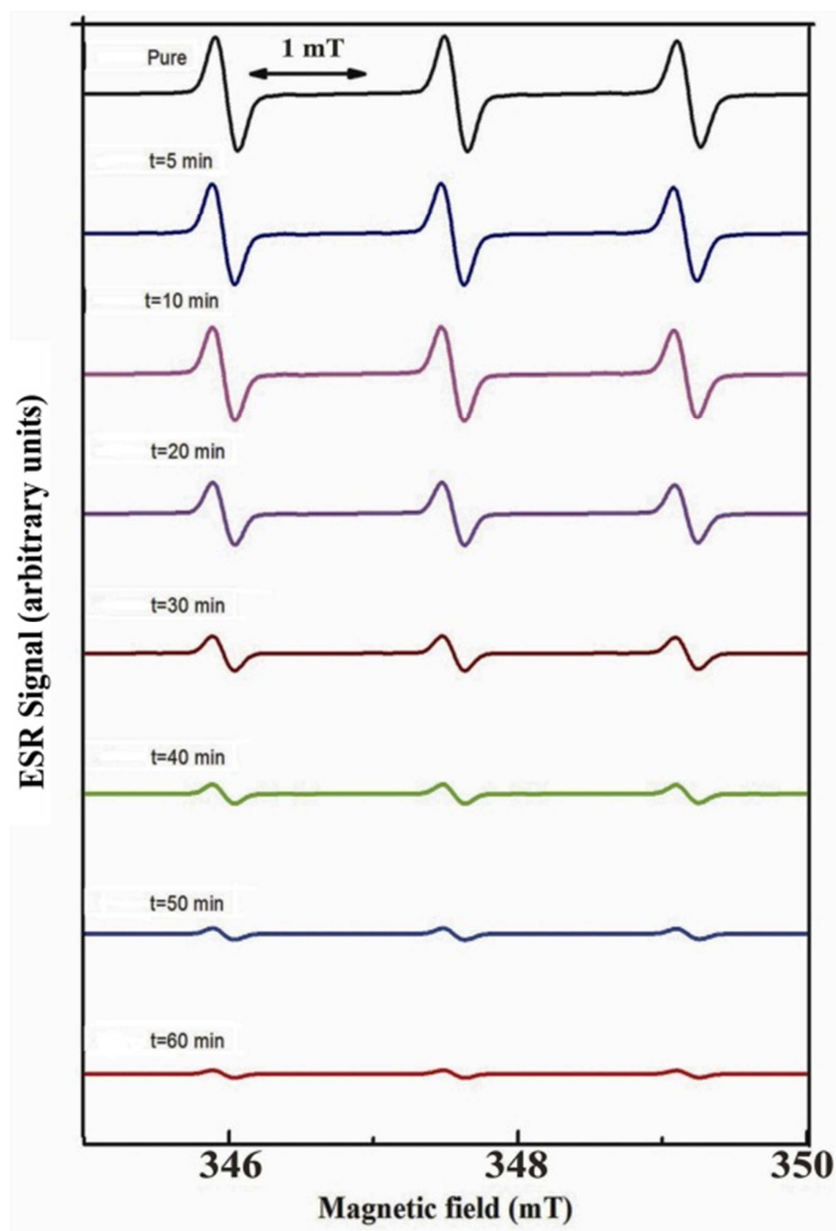

Figure 3. ESR spectra spectra of $1 \mathrm{mM}$ concentration of ${ }^{14} \mathrm{~N}$-labeled carboxy-PROXYL in pure water and $1 \mathrm{mM}$ concentration of ascorbic acid as a function of time ( $t$ ).

\subsection{Rotational Correlation Time}

The rotational correlation time describes the dynamics of the spin probe motion in the domain and it is proportional to the fluidity. The rotational correlation time is a parameter to express the mobility of spin probes in their environment. The $\tau_{\mathrm{R}}$ can be obtained from the ESR spectral line width and relative intensities. The rotational correlation time is given by an empirical formula

$$
\tau_{R}=6.5 \times 10^{-10} \Delta B_{0}\left[\left(h_{0} / h_{-1}\right)^{1 / 2}-1\right]
$$

where, $h_{0}$ and $h_{-1}$ are the heights of the central and high field line in the ESR spectrum, respectively, and $\Delta \mathrm{B}_{0}$ is the line width of the central line in Gauss [38, 39]. The rotational motion of the spin probe was assumed to be isotropic.

The rotational correlation time for $1 \mathrm{mM}$ concentration of ${ }^{14} \mathrm{~N}$-labeled carbamoyl-PROXYL, carboxy-PROXYL, methoxy-TEMPO and acetamido-TEMPO in pure water were calculated and listed in Table 2. The decrease in rotational correlation time was observed for pyrrolidine nitroxyl spin probes, carbamoyl-PROXYL and carboxy-PROXYL compared with the piperidine nitroxyl spin probes, methoxy-TEMPO and acetamido-TEMPO in pure water. These results show that the pyrrolidine nitroxyl spin probes has fast tumbling motion compared with the piperidine nitroxyl spin probes. Among the pyrrolidine nitroxyl spin probes, the carbaomyl-PROXYL has fast tumbling motion which is due to the less interaction between the carbonyl group of carbamoyl-PROXYL radical and water protons. The narrowest line width observed is an additional supporting evidence of the fast tumbling motion of the pyrrolidine nitroxyl spin probes.

\subsection{Reduction Stability}

The paramagnetic nitroxyl radical can be reduced to the corresponding hydroxylamines by reductants such as ascorbic acid. Paramagnetic nitroxyl radicals can be directly detected by ESR, while the hydroxylamines which are the reduction products of nitroxyl radicals are diamagnetic and therefore ESR silent species.

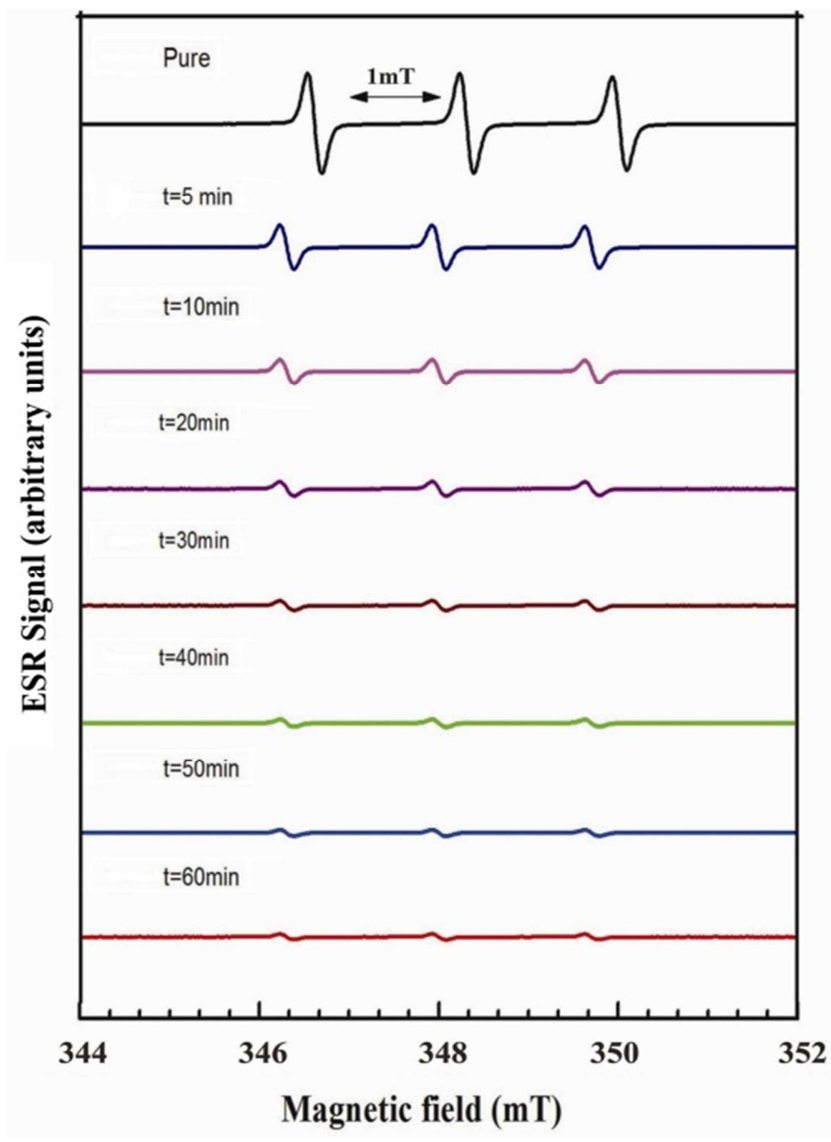

Figure 4. ESR spectra of $1 \mathrm{mM}$ concentration of ${ }^{14} \mathrm{~N}$-labeled methoxy-TEMPO in pure water and $1 \mathrm{mM}$ concentration of ascorbic acid as a function of time ( $t$ ). 
Therefore, detecting a decrease in ESR signal intensity from nitroxyl radicals due to their reduction in biological/chemical samples can be used to monitor redox invivo reactions [40, 41, 25-28]. The ESR spectrum of $1 \mathrm{mM}$ concentration of ${ }^{14} \mathrm{~N}$-labeled carbamoyl PROXYL, carboxy-PROXYL, methoxy-TEMPO and acetamido-TEMPO in pure water and $1 \mathrm{mM}$ concentration of ascorbic acid as a function of time are shown in Figures 2-5. The intensity of the ESR signal is strongly reduced in the presence of the ascorbic acid. The reduction in the signal intensity is time dependent. The reduction rate by ascorbic acid is dependent mainly upon their ring structures and substituent groups. The signal decay of several spin probes with different ring structures and substituents were compared.

\subsection{Half Life Time and the Decay Rate}

The ESR signal intensity values were fitted with a simple model of an exponential decay equation and an added constant offset, which is used to determine the half life time $\left(t_{1 / 2}\right)$ and the decay rate $(1 / \tau)$ of the nitroxyl radical in ascorbic acid $[42$, 43]. The ESR spectral intensity I ( $\mathrm{t}$ ) can be expressed as

$$
I(t)=I_{0} e^{-t / \tau}+N
$$

where $I_{0}$ is the initial value of ESR signal intensity, $t$ is the time and $1 / \tau$ is the decay rate. The decay curve for $1 \mathrm{mM}$ concentration of ${ }^{14} \mathrm{~N}$-labeled carbamoyl-PROXYL, carboxy-PROXYL, methoxy-TEMPO and acetamido-TEMPO in pure water and $1 \mathrm{mM}$ concentration of ascorbic acid was shown in Figure 6.

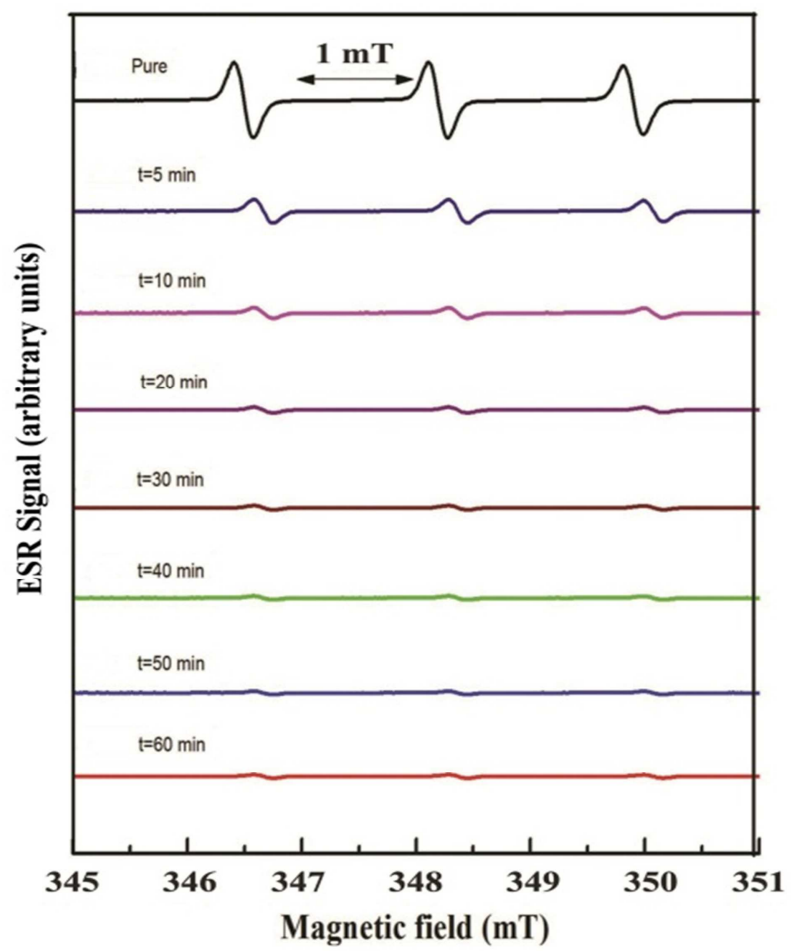

Figure 5. ESR spectra of $1 \mathrm{mM}$ concentration of ${ }^{14} \mathrm{~N}$-labeled acetamido-TEMPO in pure water and $1 \mathrm{mM}$ concentration of ascorbic acid as a function of time $(t)$.

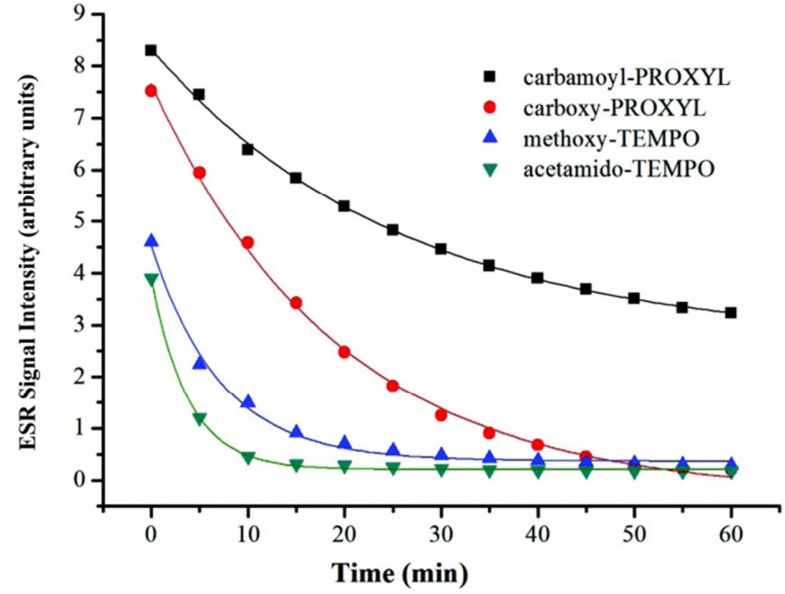

Figure 6. Time dependent decay of the ESR signal intensity for $1 \mathrm{mM}$ concentration of ${ }^{14} \mathrm{~N}$-labeled carbamoyl PROXYL, carboxy-PROXYL, methoxy-TEMPO and acetamido-TEMPO in pure water and $1 \mathrm{mM}$ concentration of ascorbic acid.

The correlation coefficient of the above curve fitting shows the best correlation $\left(\mathrm{R}^{2}>0.99\right)$. The half-life time can be calculated by using the equation,

$$
t_{1 / 2}=\tau \ln (2)
$$

The half-life time and decay rate were calculated for $1 \mathrm{mM}$ concentration of ${ }^{14} \mathrm{~N}$-labeled carbamoyl PROXYL, carboxy-PROXYL, methoxy-TEMPO and acetamido-TEMPO in $1 \mathrm{mM}$ concentration of ascorbic acid and listed in Table 3. The increase in half life time and decrease in decay rate were obtained for pyrrolidine nitroxyl spin probes compared with piperidine nitroxyl spin probes, which shows the higher stability of pyrrolidine nitroxyl spin probes. Among the pyrrolidine nitroxyl spin probes, the carbamoyl-PROXYL can act as a good permeable spin probe for invivo free radical imaging. The carboxy-PROXYL can act as a good impermeable spin probe for invivo free radical imaging.

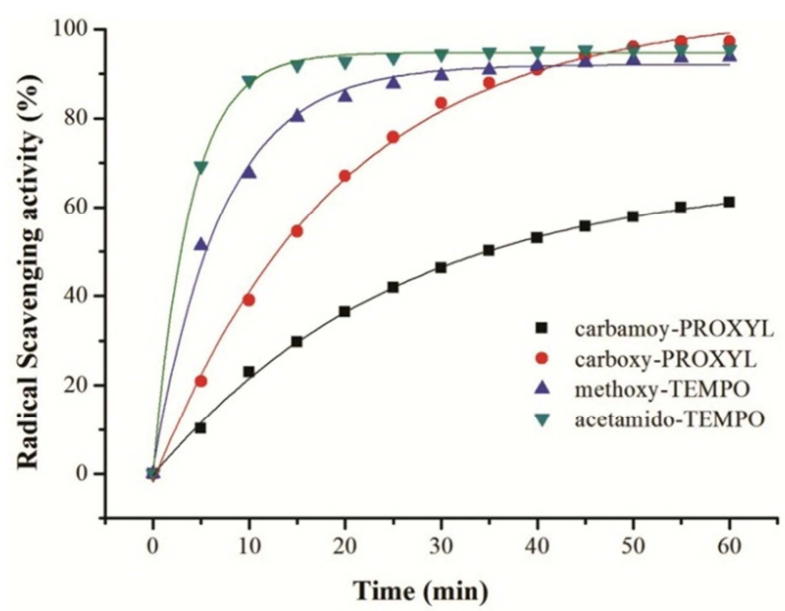

Figure 7. The radical scavenging activity (\%) of $1 \mathrm{mM}$ concentration of ${ }^{14} \mathrm{~N}$-labeled carbamoyl PROXYL, carboxy-PROXYL, methoxy-TEMPO and acetamido-TEMPO in pure water and $1 \mathrm{mM}$ concentration of ascorbic acid as a function of time. 


\subsection{Radical Scavenging Activity}

Radical scavenging activity (RSA) was expressed as the inhibition percentage of nitroxyl spin probes and calculated using the formula

$$
R S A(\%)=\frac{\left(I_{0}-I_{t}\right)}{I_{0}} \times 100
$$

where $I_{0}$ is the signal intensity of central line from the ESR spectrum, when nitroxyl radicals in pure water, $I_{t}$ is the signal intensity of central line from the ESR spectrum, when nitroxyl spin probes in ascorbic acid at time $t[44,45]$. Figure 7 shows the radical scavenging activity $(\%)$ of $1 \mathrm{mM}$ concentration of ${ }^{14} \mathrm{~N}$-labeled carbamoyl-PROXYL, carboxy-PROXYL, methoxy-TEMPO and acetamido-TEMPO in $1 \mathrm{mM}$ concentration of ascorbic acid at various time intervals. The observed radical scavenging activity is higher for pyrrolidine nitroxyl spin probes compared with the piperidine nitroxyl spin probes.

\section{Conclusions}

The ESR reduction process was recorded for nitroxyl spin probes of different ring structure with ascorbic acid as a function of time using X-band ESR spectrometer. The half-life time and decay rate were estimated. From the results, the increase in half life time and decrease in decay rate were obtained for pyrrolidine nitroxyl spin probes compared with the piperidine nitroxyl spin probes, which indicates the higher stability of pyrrolidine nitroxyl spin probes. The ESR spectra was also recorded for $1 \mathrm{mM}$ concentration of nitroxyl spin probes in pure water. The ESR parameters such as the line width, g-factor, hyperfine coupling constant and rotational correlation time were estimated. From the results, the pyrrolidine nitroxyl spin probes have narrow line width and fast tumbling motion compared with the piperidine nitroxyl spin probes. Hence, the pyrrolidine nitroxyl spin probes viz., carbamoyl-PROXYL can act as a good permeable spin probe and carboxy-PROXYL can act as a good impermeable spin probe for invivo free radical imaging. The reduction of nitroxyl spin probes could be useful as indicator of invivo redox metabolism.

\section{Acknowledgements}

The authors thank our college management for permission to carry out this work. This work was supported by the UGC Minor Research Project MRP-4314/12 (MRP/UGC-SERO).

\section{References}

[1] M. C. Krishna, S. Subramanian, P. Kuppusamy, and J. B. Mitchell, "Magnetic resonanc imaging for in vivo assessment of tissue oxygen concentration", Semin Radiat Oncol vol. 11, no. 1, pp. 58-69, 2001.

[2] K. Yamada, D. Inoue, S. Matsumoto, and H. Utsumi, "In vivo measurement of redox status in streptozotocin-induced diabetic rat using targeted nitroxyl probes", Antioxid. Redox Signal. vol. 6, no. 3, pp. 605-611, 2004.

[3] K. Kasazaki, K. Yasukawa, H. Sano, and H. Utsumi, "Non-invasive analysis of reactive oxygen species generated in $\mathrm{NH}_{4} \mathrm{OH}$-induced gastric lesions of rats using a $300 \mathrm{MHz}$ in vivo ESR technique", Free Radic. Res. vol. 37. no.7, pp 757$766,2003$.

[4] F. Hyodo, K. H. Chuang, A. G. Goloshevsky, A. Sulima, G. L. Griffiths, J. B. Mitchell, A. P. Koretsky, and M. C. Krishna, "Brain redox imaging using blood-brain barrier-permeable nitroxide MRI contrast agent", J Cereb Blood Flow Metab. vol. 28. no. 6, pp 1165-1174, 2008.

[5] K. Matsumoto, M. C. Krishna, and J. B. Mitchell, "Novel Pharmacokinetics Measurement Using Electron Paramagnetic Resonance Spectroscopy and Simulation of in Vivo Decay of Various Nitroxyl Spin Probes in Mouse Blood", The Journal Of Pharmacology And Experimental Therapeutics. vol. 310, pp 1076-1083.

[6] K. I. Yamada, P. Kuppusamy, S. English, J. Yoo, A. Irie A, S. Subramanian, J. B. Mitchell, and M. C. Krishna, "Feasibility and assessment of non-invasive in vivo redox status using electron paramagnetic resonance imaging", Acta Radiol. vol. 43, no.4, pp 433-440, 2002.

[7] A. Hirayama, K. Yoh, S. Nagase, A. Ueda, K. Itoch, N. Morito, K. Hirayama, S. Takahashi, M. Yamamoto, and A. Koyama, "EPR imaging of reducing activity in Nrf2 transcriptional factor-deficient mice", Free Radic Biol Med. vol. 34, no.10, pp 1236-1242, 2003.

[8] A. Ueda, S. Nagase, H. Yokoyama, M. Tada, H. Noda, H. Ohya, H. Kamada, A. Hirayama, and A. Koyama, "Importance of renal mitochondria in the reduction of TEMPOL, a nitroxide radicals", Mol Cell Biochem. vol. 244, no. 1-2, pp 119-124, 2003.

[9] K. Kasazaki, K. Yasukawa, H. Sona, K. Yamada, and H. Utsumi, "Application of in vivo ESR spectroscopy to pharmaceutical sciences: Evaluation of in vivo inhibitory mechanism of antigastric lesion drugs", Appl. Magn. Reson. vol. 23, no.3, pp 585-595, 2003

[10] M. Yamato, T. Egashira, and H. Utsumi, "Applications of in vivo ESR spectroscopy to measurement of cerebrovascular ROS generation in stroke", Free Radical Biology \& Medicine. vol. 35, no. 12, pp 1619-1631, 2003.

[11] K. Yamada, I. Yamamiya, and H. Utsumi, "In vivo detection of free radicals induced by diethylnitrosamine in rat liver tissue", Free Radic Biol Med. vol. 40, pp 2040-2046, 2006.

[12] Y. Kinoshita, K. Yamada, T. Yamasaki, H. Sadasue, K. Sakai, and H. Utsumi, "Development of novel nitroxyl radicals for controlling reactivity with ascorbic acid", Free Radical Research, vol. 43 no. 6, pp 565-571, 2009.

[13] A. A. Bobko, I. A. Kirilyuk, I. A. Grigor' ev, J. L. Zweier, and V. V. Kramtsov, "Reversible reduction of nitroxides to hydroxylaines: Roles for ascorbate and glutathione", Free Radical Biology \& Medicine, vol. 42, no. 3, pp 404-412, 2007.

[14] G. L. Millhauser, "Trends in Biochemical Sciences", vol. 17, no. 11, pp 448-452, 1992

[15] H. M. Swartz, and G. S. Timmins, "The metabolism of nitroxides in cells and tissues. In: Rhodes", C J., editor. Toxicology of the human environment: the critical role of free radicals. Taylor \& Francis Inc.; London, New York: 2000. pp 91-111. 
[16] O. Saphier, T. Silberstein, A. I. Shames, G. I. Likhtenshtein, E. Maimon, D. Mankuta, M. Mazor, M. Katz, D. Meyerstein, and $\mathrm{N}$. Meyerstein, "The reduction of a nitroxide spin label as a probe of human blood antioxidant properties", Free Radic. Res vol. 37 no. 3, pp 301-308, 2003.

[17] J. F. Keana, S. Pou, and G. M. Rosen, "Nitroxides as potential contrast enhancing agents for MRI application: influence of structure on the rate of reduction by rat hepatocytes, whole liver homogenate, subcellular fractions, and ascorbate", Magn. Reson. Med. vol. 5, no. 6, pp. 525-536, 1987.

[18] Z. Zhelev, R. Bakalova, I. Aoki, K. Matsumoto, V. Gadjeva, K. Anzai, and I. Kanno, "Nitroxyl Radicals for Labeling of Conventional Therapeutics and Noninvasive Magnetic Resonance Imaging of Their Permeability for Blood-Brain Barrier: Relationship between Structure, Blood Clearance, and MRI Signal Dynamic in the Brain", Molecular pharmaceutics. vol. 6, no. 2, pp. 504-512, 2009.

[19] P. Kuppusamy , H. Li, G. Ilangovan, A. J. Cardounel, J. L. Zweier, K. Yamada, M. C. Krishna, and J. B. Mitchell, "Noninvasive imaging of tumor redox status and its modification by tissue glutathione levels", Cancer Res. vol. 62, no. 1, pp. 307-12, 2002.

[20] S. M. Hahn, C. M. Krishna, A. Samuni, W. DeGraff, D. O. Cuscela, P. Johnstone, and J. B. Mitchell, "Potential use of nitroxides in radiation oncology", Cancer Res. vol. 54, pp. 2006-2010s, 1994.

[21] H. Utsumi, K. Yamada, K. Ichikawa, K. Sakai, Y. Kinoshita, S. Matsumoto, M. Nagi, "Simultaneous molecular imaging of redox reactions monitored by Overhauser-enhanced MRI with ${ }^{14} \mathrm{~N}$ - and ${ }^{15} \mathrm{~N}$-labeled nitroxyl radicals", Proc. Natl. Acad. Sci. vol. 103, pp. 1463-1468, 2006.

[22] A. Milton Franklin Benial, M. Kumara Dhas, K. Ichikawa, K. Yamada, F. Hyodo, A. Jawahar, and H. Utsumi, "Permeability Studies of Redox-Sensitive Nitroxyl Spin Probes Through Lipid Membranes Using an L-Band ESR Spectrometer", Appl Magn Reson vol. 44, no. 4, pp. 439-447, 2013.

[23] Y. Kinoshita, K. Yamada, T. Yamasaki, F. Mito, M. Yamato, N. Kosem, H. Deguchi, C. Shirahama, Y. Ito, K. Kitagawa, N. Okukado, K. Sakai, and H. Utsumi, "In vivo evaluation of novel nitroxyl radicals with reduction stability", Free Radic Biol Med. Vol. 49, no. 11, pp. 1703-1709, 2010.

[24] K. Matsumoto, F. Hyodo, A. Matsumoto, A. P. Koretsky, A. L. Sowers, J. B. Mitchell, and M. C. Krishna, "High-Resolution Mapping of Tumor Redox Status by Magnetic Resonance Imaging Using Nitroxides as Redox-Sensitive Contrast Agents", Clin Cancer Res. vol. 12, no. 8, pp. 2455-2462, 2006.

[25] G. Redler, E. D. Barth, K. S. Bauer, Jr., J. P. Y. Kao, G. M. Rosen, and H. J. Halpern, "In Vivo Electron Paramagnetic Resonance Imaging of Differential Tumor Targeting Using cis-3,4-Di(Acetoxymethoxycarbonyl)-2,2,5,5-Tetramethyl-1-P yrrolidinyloxyl”, Magn Reson Med. vol. 71, no. 4, pp. 1650-1656, 2014.

[26] H. Utsumi, K. Yasukawa, T. Soeda, K. Yamada, R. Shigemi, T. Yao, and M. Tsuneyoshi, "Noninvasive Mapping of Reactive Oxygen Species by in Vivo Electron Spin Resonance Spectroscopy in Indomethacin- Induced Gastric Ulcers in Rats", The J. Pharmacol Exp. Ther. vol. 317, no. 1, pp. 228-235, 2006.

[27] M. Yamato, T. Shiba, K. Yamada, T. Watanabe and H. Utsumi, "Noninvasive assessment of the brain redox status after transient middle cerebral artery occlusion using Overhauser-enhanced magnetic resonance imaging", Journal of Cerebral Blood Flow \& Metabolism, vol. 29, pp. 1655-1664, 2009.

[28] H. G. Fujii, H. Sato-Akaba, M. C Emoto, K. Itoh, Y. Ishihara and H. Hirata, "Noninvasive mapping of the redox status in septic mouse by in vivo electron paramagnetic resonance imaging”, Magn. Reson. Imaging. vol. 31, no. 1, pp. 130-138, 2013.

[29] M. C. Krishna, S. English, K. Yamada, J. Yoo, R. Murugesan, N. Devasahayam, J. A. Cook, K. Golman, J. H. Ardenkjaer-Larsen, S. Subramanian and J. B. Mitchell, "Overhauser enhanced magnetic resonance imaging for tumor oximetry: Coregistration of tumor anatomy and tissue oxygen concentration", Proc. Natl. Acad. Sci. USA vol. 99, no. 4, pp. 2216-2221, 2002.

[30] E. M. Gale, S. Mukherjee, C. Liu, G. S. Loving, P. Caravan, "Structure-redox-relaxivity relationships for redox responsive manganese-based magnetic resonance imaging probes", Inorg Chem. vol. 53, no. 19, pp. 10748-61, 2014.

[31] S. Matsumoto, K. Saito, Y. Takakusagi, M. Matsuo, J. P. Munasinghe, H. D. Morris, M. J. Lizak, H. Merkle, K. Yasukawa, N. Devasahayam, S. Suburamanian, J. B. Mitchell, M. C. Krishna, "In vivo imaging of tumor physiological, metabolic, and redox changes in response to the anti-angiogenic agent sunitinib: longitudinal assessment to identify transient vascular renormalization", Antioxid Redox Signal. vol. 21, no. 8, pp. 1145-55, 2014.

[32] G. Bacic, A. Pavicevic, F. Peyrot, "Invivo evaluation of different alternation of redox status by studying pharmacokinetics of nitroxide using magnetic resonance techniques", Redox Biol. doi: 10.1016/j.redox.2015.10.007, 2016.

[33] M. C. Emoto, S. Sato, H. G. Fujii, "Development of nitroxide-based theranostic compound that act both as anti-inflammatory drugs and brain redox imaging probes in MRI", Magn Reson Chem. doi: 10.1002/mrc.4431, 2016.

[34] F. Hyodo, S. Ito, K. Yasukawa, R. Kobayashi, H. Utsumi, "Simultaneous and Spectroscopic Redox Molecular Imaging of Multiple Free Radical Intermediates Using Dynamic Nuclear Polarization-Magnetic Resonance Imaging", Anal. Chem. vol. 86, pp. 7234-7238, 2014.

[35] A. Milton Franklin Benial, K. Ichikawa, R. Murugesan, K. Yamada, H. Utsumi, "Dynamic nuclear polarisation properties of nitroxyl radicals used in overhauser-enhanced MRI for simultaneous molecular imaging", J. Magn. Reson. vol. 182, pp. 273-282, 2006.

[36] A. Milton Franklin Benial, M. Kumara Dhas, A. Jawahar, "Rotational correlation time studies on nitroxyl radicals using $300 \mathrm{MHz}$ ESR spectrometer in high viscous liquid", Appl Magn Reson. vol. 40, pp. 311-319, 2011.

[37] I. Nicholson, D. J. Lurie and F. J. L. Robb, "The applications of proton electron double resonance imaging technique to proton mobility studies", J. Magn. Reson. Ser. B, vol. 104, pp. 250-255, 1994

[38] P. F. Knowles, D. Marsh, H. W. E. Rattle, An Introduction to the Theory and Practice of NMR and ESR in Biological Systems Magnetic Resonance of Bio-molecules: An Introduction to the Theory and Practice of NMR and ESR in Biological Systems, Wiley, London, 1976. 
[39] M. J. G. W. Roozen and M. A. Hemminga, "Molecular motion in sucrose-water mixtures in the liquid and glassy state as studied by spin probe ESR". J. Phys. Chem. vol. 94 no. 19, pp. 7326-7329, 1990 .

[40] F. Yoshino, A. Yoshida, N. Umigai, K. Kubo and M. C. Lee, "Crocetin reduces the oxidative stress induced reactive oxygen species in the stroke prone spontaneously hypertensive rats (SHRSPs) brain" J. Clin. Biochem. Nutr. vol. 49, no. 3, pp. 182-187, 2011.

[41] K. Takeshita, K. Kawaguchi, K. Fujii-Aikawa, M. Ueno, S. Okazaki, M. Ono, M.C. Krishna, P. Kuppusamy, T. Ozawa, and N. Ikota, "Heterogeneity of Regional Redox Status and Relation of the Redox Status to Oxygenation in a Tumor Model, Evaluated Using Electron Paramagnetic Resonance Imaging”, Cancer Res. vol. 70, no. 10, pp. 4133-4140, 2010.

[42] G. L. Caia, O. V. Efimova, M. Velayutham, M. A. El-Mahdy, T. M. Abdelghany, E. Kesselring, S. Petryakov, Z. Sun, A.
Samouilov and J. L. Zweier, "Organ specific mapping of in vivo redox state in control and cigarette smoke-exposed mice using EPR/NMR co-imaging”, J. Magn.Reson. vol. 216, pp. 21-27, 2012.

[43] R. M. Davis, S. Matsumoto, M. Bernardo, A. Sowers, K. Matsumoto, M. C. Krishna and J. B. Mitchell, "Magnetic resonance imaging of organic contrast agents in mice: capturing the whole-body redox landscape", Free Radic. Biol. Med. vol. 50, pp. 459-468, 2011.

[44] R. Amarowicz, R. B. Pegg, P. Rahimi-Moghaddam, B. Barl and J. A. Weil, "Free-radical scavenging capacity and antioxidant activity of selected plant species from the Canadian prairies", Food Chemistry. vol. 84, pp. 551-562, 2004.

[45] S. Mathew and T. E. Abraham, "In vitro antioxidant activity and scavenging effects of cinnamomum verum leaf extract assayed by different methodologies", Food Chem. Toxicol. vol. 44, pp. 198-206, 2006. 